\title{
Imported Malaria
}

\section{Stela Halichidis*}

Clinical Hospital of Infectious Diseases, Constanta, Romania

Malaria is a parasitic disease caused by different species of Plasmodium (Plasmodium falciparum, Plasmodium vivax, Plasmodium ovale, Plasmodium malariae and Plasmodium knowlesi), transmitted by the Anopheles mosquitoes, so the condition for the disease is the existence of this vector. Because of this, the prevalence is high only in some endemic areas, where are the predisposing conditions for these vectors. That's why in Europe, usually imported cases are registered. In some studies, many cases from Europe were registered in travellers from Africa, South America, Asia and only a few in the Middle East. The inadequate prophylaxis used for tourists and navigators in endemic areas was the principal cause of the mentioned cases. Prophylaxis must be done for all travellers in endemic areas [1].

The symptoms and signs of malaria are not specific, so the epidemiological inquest, and, in particular, the travel history can be useful for the diagnosis in patients with fever. For non-immune patients, the diagnosis and treatment are medical emergencies, because the disease is more severe than in those from endemic areas [2]. Usually, the most severe forms are caused by infections with P. falciparum and the incubation period is at around 30 days, but this period can be longer in those with incomplete malaria chemoprophylaxis, that can reduce the parasites in the blood, but the protection is not provided. That's why, some studies noticed a longer than usual incubation period, for 47 days and, in one case, for 120 days after leaving Angola [3]. The diagnosis is based on stained thick and thin blood films; rapid tests are also available but can be useful only for sceening, every suspicion must be checked with the microscopy method.

In common forms of Plasmodium falciparum malaria, the treatment consist of artemisinin or the combination atovaquone/proguanil. In Europe, artemether/lumefantrine and dihydroartemisinin/ piperaquine are available. The other species of malaria (Plasmodium vivax, Plasmodium ovale, Plasmodium malariae and Plasmodium knowlesi), can also be sensible on artemisinin combination therapy, but these species can be treated with chloroquine. For the treatment with primaquine for liver forms with P. vivax and P. ovale, we have to exclude the glucose 6-phosphate dehydrogenase deficiency.

There are some specific features in malaria's treatment depending of the patient's condition: age of the patient, pregnancy and associated diseases or therapy [2]. Imported malaria with P. falciparum can be fatal [4] and the election treatment consists of intravenous artesunate [5] that reduce the parasitaemia soon, compared to quinine. A Cochrane review concluded that treatment with artesunate significantly reduced the risk of death both in adults (RR 0.61, 95\% Confidence Interval (CI) 0.50 to $0.75 ; 1664$ participants, five trials) and children (RR $0.76,95 \%$ CI 0.65 to $0.90 ; 5765$ participants, four trials) [6].

Anyway, the antimalarial treatment is not simple, because of the possible serious side-effects, or of the complications of the disease, so the patients, especially those outside the endemic areas, without protectors (antibodies) must be closely monitored, some in ICU, during the acute stage, but they also require long-term surveillance after the disease.

\section{References}

1. Rugina S, Lungu CS, Halichidis S, Rugina CN (1998) Imported Malaria in Romania. Analysis about 76 cases. XXVth Balkan Medical Week 15: 283.

2. Helena HA, Bruneel F, Burchard G, Castelli F, Chiodini P, et al. (2012) Management of imported malaria in Europe. Malaria Journal 11: 328.

3. Santos LC, Abreu CF, Xerinda SM, Tavares M, Lucas R, et al. (2012) Severe imported malaria in an intensive care unit: a review of 59 cases. Malar J 11: 96

4. Société de Pathologie Infectieuse de Langue Francaise, College des Universitaires de Maladies Infectieuses et Tropicales, Société Française de Médecine des Armées, Société Française de Parasitologie, Société Française de Pédiatrie, et al. (2008) Recommendations for clinical practice. Management and prevention of imported Plasmodium falciparum malaria. Med Mal Infect 38:54-67.

5. World Health Organization (2010) World Malaria Report 2010. WHO, Geneva.

6. Sinclair D, Donegan S, Lalloo DG (2011) Artesunate versus quinine for treating severe malaria. Cochrane Database Syst Rev 16. 